\title{
A Weakest Precondition Semantics for an Object-Oriented Language of Refinement
}

\author{
Ana Cavalcanti ${ }^{1}$ and David A. Naumann ${ }^{2}$ \\ 1 Departamento de Informática \\ Universidade Federal de Pernambuco, Po Box 7851 50740-540 Recife PE Brazil \\ Phone: +55 812718430 Fax: +55 812718438 \\ alcc@di.ufpe.br www.di.ufpe.br/ alcc \\ 2 Department of Computer Science \\ Stevens Institute of Technology, Hoboken NJ 07030 USA \\ naumann@cs.stevens-tech.edu www.cs.stevens-tech.edu/ naumann
}

\begin{abstract}
We define a predicate-transformer semantics for an objectoriented language that includes specification constructs from refinement calculi. The language includes recursive classes, visibility control, dynamic binding, and recursive methods. Using the semantics, we formulate notions of refinement. Such results are a first step towards a refinement calculus.
\end{abstract}

Keywords: refinement calculi, semantic models, object-orientation, verification

\section{Introduction}

There has been extensive study of formal type-systems for object-oriented languages, and some study of formal specification, but formalization of development methods [BKS98, Lan95] lags behind both the language features and the informal methods presently used. This paper presents a semantic basis for formal development of programs in languages like Java and $\mathrm{C}++$. Our language, called ROOL (for Refinement Object-oriented Language), is sufficiently similar to Java to be used in meaningful case studies and to capture some of the central difficulties, yet it is sufficiently constrained to make it possible to give a comprehensible semantics.

We assume the reader is familiar with basic concepts and terminology of object-oriented programming. We address the following challenging issues.

- Dynamic binding of methods means that the version of a method that will be invoked is determined only at run time. Such programs exhibit phenomena similar to higher-order imperative programs.

- Classes are important in practice for modularity, but they are complicated to model (for which reason many studies focus on instance-oriented subtyping).

- Object-oriented programs involve fine-grained control of visibility in terms of private, inherited, and public identifiers.

Our language has mutually recursive classes and recursive methods. We omit reference types, however. Pointers are ubiquitous in practice, but so are techniques 
to isolate deliberate sharing from the many situations where value semantics is preferable. Our object values are tuples with recursive nesting but no sharing. We leave pointers as an important but separate issue AdB94.

Our work is part of a project that aims to extend to object-oriented programming the most widely-used and well-developed formal methods - those associated with Hoare logic and weakest preconditions. Because behavioural subclassing involves intermingled programs and specifications [LW94, it is natural to extend a refinement calculus Mor94 BvW98. As usual in refinement calculi, our semantics is based on weakest preconditions.

In the approach we adopt, commands denote functions on formulas. In isolation, purely syntactic transformer semantics is dubious. While our collaborators are developing a state-transformer semantics which will make it possible to prove operational soundness, we have taken the preliminary step of giving a set-theoretic semantics for predicate formulas and expressions, for the typecorrectness results. Object states are represented by tuples of attribute values, and in general types denote sets of values. Methods are treated as procedures with a distinguished self parameter. Classes denote tuples of method meanings. Predicate formulas denote sets of states. The interplay between the valueoriented semantics of expressions and the formula-oriented semantics of commands is mediated by the semantics of formulas.

The semantics is based on a typing system. In the methodological literature simpler approaches are usually taken: there is a fixed global typing, or untyped variables are used and types are treated as predicates. A fixed global typing is unsuitable for formulating laws about subclasses and inheritance; and treating types as predicates risks inconsistency in the presence of higher-order phenomena. We employ techniques that have become standard in type theory and denotational semantics; the semantics is defined in terms of typing derivations, which provides convenient access to necessary contextual information.

We do not treat more advanced notions of subtyping than those in Java: we are interested in reasoning about type casts and tests as they are used in Java and its cousin languages. The typing system is similar to that of $\mathrm{Nau} 98 \mathrm{~b}$, and also to those used in typing algorithms, where subsumption is incorporated into rules for different constructs rather than being present as a general rule. Nonetheless, soundness of our definitions is not at all obvious, due to subtleties of modelling dynamic binding as well as mutually recursive classes. In this paper, we disallow mutually recursive methods, which lets us use a simpler, though non-trivial, well-ordering to show the semantics is well defined.

The main contribution of this paper is the extension of standard weakest precondition semantics to a Java-like language with classes, visibility, dynamic binding, and recursion. We give basic results on soundness of the definitions, define notions of program and class refinement, and show that the constructors of ROOL are monotonic with respect to refinement. Our semantics is being used in ongoing research on practical specification and verification. For reasons of space we omit many definitions and proofs that appear in CN99. 


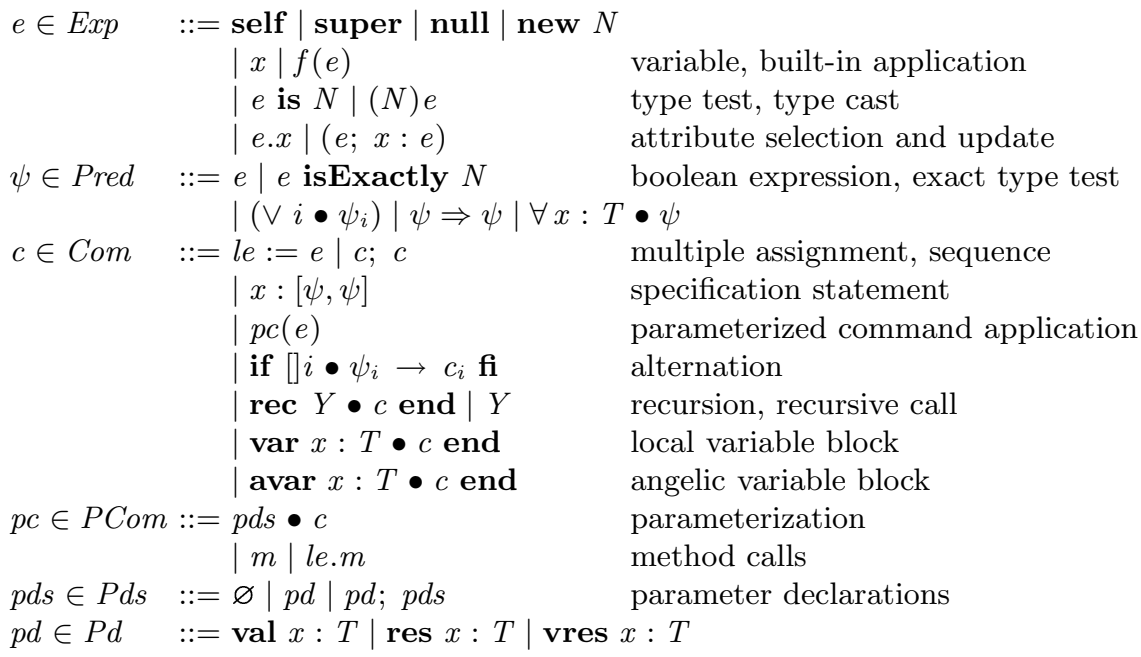

Table 1. Expressions, selected predicates, commands, and parameterized commands.

\section{Language}

The imperative constructs of ROOL are based on the language of Morgan's refinement calculus [Mor94], which extends Dijkstra's language of guarded commands. Specifications are regarded as commands; we use the word command to refer to specifications, commands in the traditional sense, and hybrids where programming structures and specifications are mixed.

Data types $T$ are the types of attributes, local variables, method parameters, and expressions. They are either primitive (bool, int, and others) or class names $N$. Primitives may include functional types such as arrays of integers.

The expressions $e$ are generated by a rule in Table 11. We assume that $x$ stands for a variable identifier, and $f$ for a literal or built-in function. Built-ins should include primitive predicates like equality. The update $\left(e_{1} ; x: e_{2}\right)$ denotes a fresh object copied from $e_{1}$ but with the attribute $x$ mapped to a copy of $e_{2}$. Attribute selection $e . x$ is a run-time error in states where $e$ denotes null, and $(N) e$ is an error if the value of $e$ is not of dynamic type $N$. The type test $e$ is $N$ checks whether non-null $e$ has type $N$; it is false if $e$ is null, like instanceof in Java. The predicates $\psi$ of ROOL include formulas of first-order logic, program expressions of type bool, and exact type tests $e$ isExactly $N$.

We identify a subset Le of Exp; these left-expressions can appear as the target of assignments and method calls, and as result and value-result arguments.

$$
l e \in L e::=l e 1 \mid \text { self } \mid \text { self.le } 1 \quad \text { le } 1 \in L e 1::=x \mid \text { le } 1 . x
$$

Assignments to self and method calls with self as a result or value-result argument would never appear in user programs, but they are used in the seman- 
tics. We allow $l e$ in $l e:=e$ to range over finite non-empty sequences of leftexpressions, and $e$ over corresponding lists.

For alternation we use an informal indexed notation for finite sets of guarded commands. Specification statements are as in [Mor94. Methods are defined using procedure abstractions in the form of Back's parameterized commands val $x: T \bullet c$, res $x: T \bullet c$, or vres $x: T \bullet c$ CSW99]. These correspond to parameter passing by copy: call-by-value, by-result, and by-value-result, respectively. In each case, $x$ stands for a finite sequence of variable identifiers, $T$ for a corresponding list of types, and $c$ for a command. We use $x$ to stand both for a single variable and for lists of variables; the context should make clear which one is meant. The same comment applies to our uses of $e$ and $T$.

A parameterized command can be applied to a list of arguments to yield a command. A method call is a parameterized command. A call $m$ refers to a method of the current object; a call le. $m$ refers to a method associated with the object that is the current value of $l e$. We do not allow method calls $e_{1} \cdot m(e)$ where $e_{1}$ is a general expression, because it is convenient in the semantic definitions that the object is named by a variable (the same is done in AL97]). If $e_{1}$ is not a left-expression, $e_{1} \cdot m(e)$ is equivalent to var $x: T \bullet x:=e_{1} ; x . m(e)$ end, where $x$ is fresh. This is not an adequate replacement for $e_{1} \cdot m(e)$, when $e_{1}$ is a left-expression, because it does not make persistent the changes to $e_{1}$. However, calls of the form le.m(e) are available in ROOL.

A program is a sequence of class declarations followed by a command.

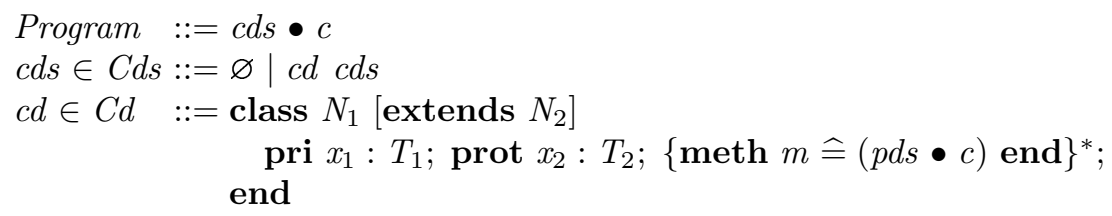

A class declaration $c d$ introduces a class named $N_{1}$. The optional extends-clause determines the immediate superclass of $N_{1}$. In its absence, $N_{1}$ extends object, which has no attributes or methods. The pri and prot clauses introduce the private and protected attributes of $N_{1}$ (recall that $x_{1}$ and $x_{2}$ can be lists). The visibility mechanism is similar to that of Java: private attributes are visible just inside the class, and protected attributes are visible in the class and in its subclasses. Following the pri and prot clauses, there is a list of method declarations. The method introduced by meth $m \widehat{=}(p d s \bullet c)$ end is named $m$; its body is the parameterized command $(p d s \bullet c)$. All methods are considered to be public.

\section{Typing}

Besides the data types $T$, other phrase types $\theta$ are available for predicate formulas, commands, parameterized commands, and complete programs.

$$
\theta::=T|\operatorname{pred}| \operatorname{com}|\operatorname{pcom}(p d s)| \operatorname{program}
$$


The types of phrases, in the context of a collection of class declarations, a specific class, and some method parameter and local variable declarations, are given by the typing relation $\triangleright$. For example, $\Gamma, N \triangleright c$ : com asserts that $c$ is a command that can appear in the body of a method in class $N$. Here $\Gamma$ is a typing environment; it records class declarations as well as locals for $c$ : the attributes visible in $N$, and method parameters and local variables in scope. Similarly, $\Gamma, N \triangleright e: T$ asserts that in a method of $N, e$ is an expression of type $T$.

\subsection{Typing Environment}

We assume the existence of two disjoint sets of names: the set CName of class names and the set LName of local names. A local may be either an attribute, a method, a method parameter, or a local variable. We also distinguish two class names: object and main. The former is the superclass of all classes. The latter does not refer to a class itself, but to the main part of a complete program.

A typing environment $\Gamma$ is a record with six fields: attr, meth, vis, cnames, supcls, and locals. The first, attr, is a finite partial function CName $\rightarrow$ LSignature. An LSignature associates a local name with a type: LSignature = LName $\rightarrow$ Type. The field attr records the names and types of all declared and inherited attributes of every declared class. Similarly, meth records the names and signatures of all declared and inherited methods of the known classes: meth has type CName $\pitchfork$ MDecs where MDecs $=$ LName $\pitchfork$ Pds.

The third field of a typing environment, vis, records the visibility of the attributes of the declared classes: vis has type CName $\pitchfork($ LName $\pitchfork$ Visibility) where Visibility $=\{$ pri, prot, $i p r i\}$. If, we have that for an attribute $x$ of a class $N$, vis $N x=$ pri, then $x$ is a private attribute of $N$ that was declared (and not inherited) by $N$; the inherited private attributes of $N$ are associated to ipri. Finally, prot refers to the protected (either inherited or declared) attributes.

The cnames field of a typing environment is a set containing the name of all declared classes: cnames $=$ dom attr $=$ dom meth $=$ dom vis. The distinguished class name object is supposed to be in cnames, while main, which does not refer to a class, is supposed not to be in cnames. Moreover, the class object is associated to the empty signature in both attr and meth.

The supcls field of a typing environment associates a class name to the name of its immediate superclass: supcls has type CName $\leftrightarrow$ CName. All declared classes have a direct superclass: either a class mentioned explicitly in their declarations or object. On the other hand, object itself does not have a superclass. Furthermore, a superclass is a declared class and the inheritance relationship is not allowed to have circularities. The subtype relation $\leq_{\Gamma}$ is defined by $T_{1} \leq_{\Gamma} T_{2} \Leftrightarrow\left(T_{1}, T_{2}\right) \in(\text { I.supcls })^{+} \vee T_{1}=T_{2}$.

The last component of a typing environment, locals, is an LSignature that records the types of the visible atributes of the current class, and of any method parameter and local variables in scope. The attributes are also recorded in attr; this redundancy simplifies typing rules. The classes referred to in the signatures in the range of either attr or meth and in locals must be declared. 


$$
\begin{aligned}
& \frac{N \neq \text { main }}{\Gamma, N \triangleright \text { self }: N} \quad \frac{N^{\prime} \in \Gamma \text {.cnames }}{\Gamma, N \triangleright \text { new } N^{\prime}: N^{\prime}} \quad \frac{\Gamma, N \triangleright e: N^{\prime} N^{\prime \prime} \leq_{\Gamma} N^{\prime}}{\Gamma, N \triangleright e \text { is } N^{\prime \prime}: \text { bool }} \\
& \frac{\Gamma, N \triangleright e: N^{\prime} N^{\prime \prime} \leq_{\Gamma} N^{\prime}}{\Gamma, N \triangleright\left(N^{\prime \prime}\right) e: N^{\prime \prime}} \quad \frac{\Gamma, N \triangleright e: N^{\prime} \quad \Gamma \text { attr } N^{\prime} x=T \quad \text { visib } \Gamma N^{\prime} N x}{\Gamma, N \triangleright e . x: T} \\
& \frac{\Gamma \triangleright e: \text { bool }}{\Gamma \triangleright e: \text { pred }} \quad \frac{\Gamma \triangleright \psi_{i}: \text { pred for all } i}{\Gamma \triangleright\left(\vee i \bullet \psi_{i}\right): \text { pred }} \quad \frac{\Gamma ; x: T \triangleright \psi: \text { pred }}{\Gamma \triangleright \forall x: T \bullet \psi: \text { pred }} \\
& \frac{\Gamma, N \triangleright e: N^{\prime} N^{\prime \prime} \leq_{\Gamma} N^{\prime}}{\Gamma, N \triangleright e \text { isExactly } N^{\prime \prime}: \text { pred }} \quad \frac{\left(\Gamma ; x: N^{\prime \prime}\right) \triangleright \psi: \text { pred } N^{\prime \prime} \leq_{\Gamma} N^{\prime}}{\left(\Gamma ; x: N^{\prime}\right) \triangleright x \text { isExactly } N^{\prime \prime} \wedge \psi: \text { pred }}
\end{aligned}
$$

Table 2. Typing of selected expressions and predicates.

A typing $\Gamma, N \triangleright$ phrase : $\theta$ holds just if it is well formed and is derivable using the rules to be presented in the sequel. Well formedness is characterised by three properties. First, $\Gamma$ has to satisfy the conditions above for environments. Secondly, the current class must be declared: $N \neq$ main $\Rightarrow N \in \Gamma$.cnames. Thirdly, dom $\Gamma$.locals should include all visible attributes of $N$, i.e. the declared private and the declared and inherited protected attributes - all but the inherited private ones. We assume that no parameter or local variable has the same name as an attribute of the class. If $N$ is main there are no restrictions on $\Gamma$.locals, which contains only parameters and local variables.

\subsection{Expressions and Predicates}

Typing rules for some expressions and predicates are in Table 2] The boolean expression $e$ is $N^{\prime \prime}$ is well-typed when the type of $e$ is a superclass of $N^{\prime \prime}$. The type of $e . x$ is that of the $x$ attribute of the class of $e$, provided this attribute is visible from the current class. In a hypothesis like $\Gamma$ attr $N^{\prime} x=T$, which involves partial functions, we mean that the expressions are defined and equal. Visibility is considered in visib $\Gamma N^{\prime} N x$, a condition stating that, according to $\Gamma, x$ is an attribute of $N^{\prime}$ visible from inside $N$. We define visib $\Gamma N^{\prime} N x$ to hold if and only if $N \leq_{\Gamma} N^{\prime}$, .vis $N x \neq i$ pri, and $N \neq N^{\prime} \Rightarrow$ T.vis $N x \neq$ pri. The attributes visible in $N$ are those declared in $N$ itself and those inherited from its superclasses that are not private.

A typing $\Gamma, N \triangleright \psi$ : pred is for a predicate on the state space of a method in class $N$, where $\Gamma$.locals declares local variables, parameters, and attributes to which $\psi$ may refer. We say $\psi$ is typable in $\Gamma, N$, meaning $\Gamma, N \triangleright \psi$ : pred is derivable; similarly for command typings later. In some rules we omit the current class $N$ because it does not change throughout the rule. The environment $\Gamma ; x: T$, differs from $\Gamma$ just in the locals field: we define $(\Gamma ; x: T)$.locals to be $\Gamma$.locals $\oplus\{x \mapsto T\}$, where $\oplus$ denotes function overriding.

The rule for isExactly is similar to the rule for is, but we also need coercion rules for is and isExactly in combination with $\wedge$ and $\Rightarrow$. As an example, consider 


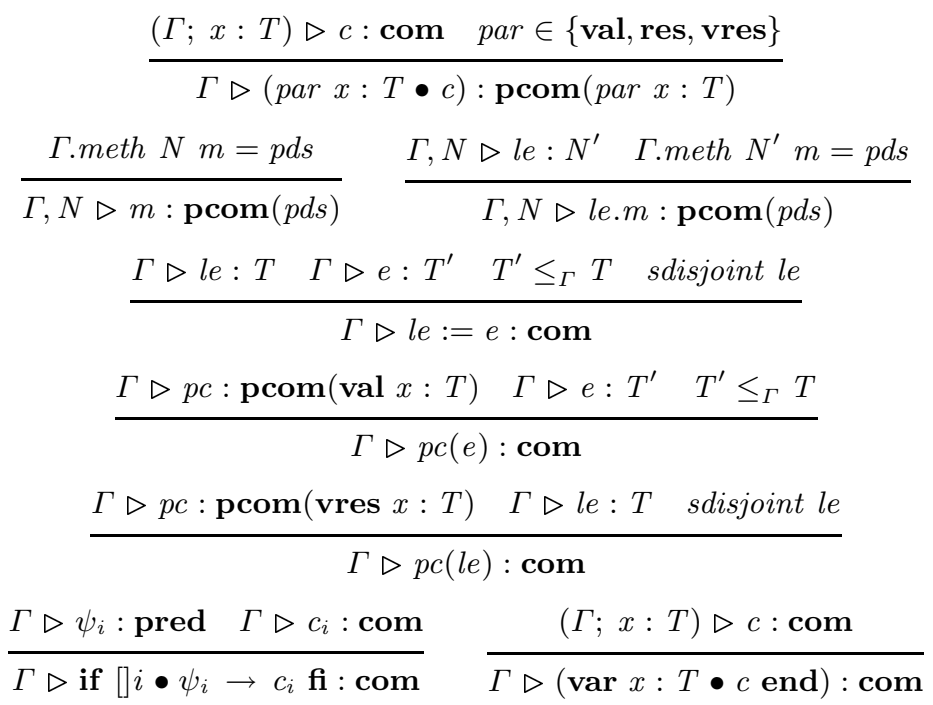

Table 3. Typing of selected parameterized commands and commands.

a class $P t$ of points and an extended class $C p t$ with an added attribute color. The predicate $(x$. color $=$ red $)$ is not typable in a context $(\Gamma ; x: P t), N$. However, if for instance $(\Gamma, x: C p t), N \triangleright x$. color $=$ red $:$ pred, we would like the predicate $x$ is $C p t \Rightarrow x . c o l o r=$ red to be typable in a context where $x$ has type Pt. Using only the separate rules for is and $\Rightarrow$, it is not typable as such; but it can be typed by a coercion rule for is like the one for isExactly in Table 2, Rules like this allow the derivation of typings in more than one way, but the semantic definitions ensure that the meaning is independent of derivation (Lemma 6).

Substitution on formulas and expressions is standard, but it is worth noting that the free variables of $e . x$ are those of $e$. This is because $x$ is in the role of an attribute name.

\subsection{Parameterized Commands, Commands, and Programs}

Typing rules for selected commands and parameterized commands are presented in Table 3. The type of a parameterized command records its parameter declarations. In the cases of $m$ and le. $m$, the declarations are recorded in the meth attribute of the typing environment. Of course, le.m is well-typed only if the type of le is a class with a method $m$. An omitted rule deals with multiple parameters.

To preclude aliasing, the rule for assignment stipulates sdisjoint le. This means that, if $l e$ is a list, then no member of le is a prefix of another, after deleting self. For example, neither $x, x . y$ nor $x$, self. $x$ is sdisjoint, but $x, y . x$ is. If $p c$ is a parameterized command with parameter declaration val $x: T$, then $p c(e)$ is well-typed when the type of $e$ is a subtype of $T$. If $x$ is a result or a 
value-result parameter, then $p c$ can only be applied to sdisjoint left-expressions. If $x$ is a result parameter, $p c(l e)$ is well-typed when $T$ is a subtype of the type of le. When $x$ is a value-result parameter, these types have to be the same.

A complete program $c d s \bullet c$ is well-typed in an environment where only global variables $x$ are in scope, just when $c$ is well-typed in the environment $\Gamma$ determined by $c d s$ and $x: T$, and considering that the current class is main.

$$
\begin{gathered}
\Gamma, \text { main } \triangleright c: \text { com } \quad \Gamma=((\text { VDecs } c d s \text { main }) ; x: T) \\
\text { Vmeth } \Gamma c d s \quad \text { nomrec } \Gamma \text { cds }
\end{gathered}
$$

$(\varnothing ; x: T) \triangleright c d s \bullet c:$ program

The fields of the environment $\varnothing$ are all empty, so that in $(\varnothing ; x: T)$ the only nonempty field is locals, which records the global variables $x: T$ of the program. The function VDecs extracts information from and checks a sequence of class declarations. In the environment determined by this function, the classes are associated with both its declared and inherited methods. The condition Vmeth $\Gamma \mathrm{cds}$ checks that the method bodies in $c d s$ are well-typed in the environment $\Gamma$. The method bodies are checked in an environment that includes their signatures, so recursive calls are appropriately dealt with. Mutually recursive calls, however, are not allowed. This is verified by the condition nomrec $\Gamma \mathrm{cds}$.

The absence of mutual recursion between methods can not be checked as easily as the absence of mutual recursion between procedures of a traditional imperative program. By way of illustration, consider classes $C, D$ and $C^{\prime}$; the class $C$ has an attribute $a$ of type integer and a method $m 1$ that, for instance, increments $a$ by 1 . The class $D$ has an attribute $c$ of class $C$, a method $m 2$ with a call c. $m 1()$, and some other methods. There is no mutual recursion, as $m 1$ does not call $m 2$. However, suppose that in a subclass $C^{\prime}$ of $C$ we declare an attribute $d: D$ and redefine $m 1$ introducing a call $d . m 2()$. Now, if the private attribute $c$ of $D$ happens to have dynamic type $C^{\prime}$ when $m 2$ is called, then mutual recursion will arise. To rule out mutual recursion, we require that if a method $m 2$ calls a method $m 1$ then neither $m 1$ nor any of its redefinitions calls $m 2$.

\subsection{Properties of Typing}

To a large extent, a context determines the type of an expression; an exception is null, for which we have $\Gamma, N \triangleright$ null : $N^{\prime}$ for all $N, N^{\prime}$. Some phrases, however, can be typed in many contexts. For example, considering again the class $P t$ and its subclass $C P t$, the command $x:=$ new $C P t$ can be typed in $\Gamma ; x: P t$ and also in $\Gamma ; x: C P t$. Nonetheless, an expression typing does determine a derivation.

Lemma 1. For all typings $\Gamma, N \triangleright e: T$, there is at most one derivation.

For predicates, the coercion rules make it possible to derive certain typings in more than one way. For example, if $\psi$ is derivable in $\left(\Gamma ; x: N^{\prime}\right), N$, then $\left(\Gamma ; x: N^{\prime}\right), N \triangleright x$ is $N^{\prime} \Rightarrow \psi:$ pred can be derived using the rules for is and $\Rightarrow$, or using a coercion rule; more on this later.

To show type-correctness of method calls we need the following result. It is similar to the coercion rules, but in fact it does not depend on them. 
Lemma 2. The following rule is admissible, in the sense that the conclusion is derivable if the hypothesis are.

$$
\Gamma, N \triangleright \psi_{N^{\prime}} \text { : pred for all } N^{\prime} \leq_{\Gamma} N \quad N \neq \text { main }
$$

$\Gamma, N \triangleright\left(\vee_{N^{\prime} \leq{ }_{\Gamma}} \bullet\right.$ self isExactly $\left.N^{\prime} \wedge \psi_{N^{\prime}}\right)$ : pred

Many type systems include a rule of subsumption, but this would make coherence (Lemma 6) harder to prove. The useful effects of subsumption are built-in to the typing rules.

\section{Semantics}

Since ROOL includes infeasible (discontinuous) constructs, recursive class definitions cannot be interpreted by standard domain-theoretic techniques. We deal with recursive classes by separating attributes from methods, so the domain equations to be solved are simple "polynomials" involving first-order records.

The semantics $\llbracket \Gamma, N \triangleright$ phrase : $\theta \rrbracket$ of each derivable typing, except method call, is defined as a function of the semantics of its constituent phrases. Most typing rules have a corresponding semantics which we present in a form that mimics the typing rule, to remind the reader of the typings for constituent phrases and any side conditions on those typings. Some phrases are treated indirectly through syntactic transformations described later.

Method calls are the most complicated part of the semantics, and they are discussed last. Semantics of method call goes beyond recursion on typing derivations. Moreover, we need the semantics to be defined for any phrase typable in an extended typing system defined as follows. The first change is that constraints involving the predicate visib are dropped. The second is that, in the rules for type tests and type casts, the subtyping constraint is dropped.

Semantically, $e$ is $N^{\prime \prime}$, for example, can only hold if $N^{\prime \prime}$ is a subtype of the declared type of $e$. Nevertheless, this constraint is incompatible with the semantics of assignment, which as usual is interpreted by substitution. Consider, for instance, a context $\Gamma$ with locals $x: P t, z: S C P t$ where $S C P t \leq_{\Gamma} C P t \leq_{\Gamma} P t$. In this context, both $x:=z$ and $x$ is $C P t$ are typable, but substitution yields $z$ is $C P t$ which is not typable in the original system because $C P t \not_{\Gamma} S C P t$.

All results in Section 3.4 hold for both typing systems. The constraints we drop are natural for user programs, but such constraints are not found in semantic studies. Although user specifications would not refer to non-visible attributes, such predicates can be used in proofs of laws.

\subsection{Environments, Data Types, and States}

An environment is a finite partial function CName $\rightarrow$ (LName $\rightarrow$ PCom) that for a given class associates method names to parameterized commands. As formalized later on, the parameterized command corresponding to a method will be that given in its declaration, with an extra parameter me. This parameter 
is passed by value-result and provides the attributes of the object upon which the method is called. This facilitates interpretation of the method body in the context of its calls.

For a given typing environment $\Gamma$, we define the set $\llbracket \Gamma \rrbracket$ of environments compatible with $\Gamma$. The environments $\eta$ in $\llbracket \Gamma \rrbracket$ are characterized by the following conditions. First, dom $\eta=\Gamma$.cnames. Also, $\operatorname{dom}(\eta N)=\operatorname{dom}($ I.meth $N)$ for all $N \in \operatorname{dom} \eta$. Finally, the parameter declarations are those recorded in $\Gamma$.meth, along with the extra value-result parameter $m e$; for all $N, m$ there is some $c$ such that $\eta N m=($ vres me $N$; .meth $N m \bullet c)$. In the environments we construct later, $c$ is derived from the declared body as a fixpoint.

In addition to the environment, the semantic function for expressions also takes a state as argument. A state assigns type-correct values to the attributes of the current object, and to the parameters and local variables. It also records the class of the current object. Object values, like states, assign values to attribute names. Our formalization begins with a universal set of untyped values, which are then used for the semantics of specific data types and state types.

The sets Value and ObjValue are the least solutions to the equations below. We assume the unions are disjoint. The symbol $\triangleleft$ means domain subtraction.

$$
\begin{aligned}
& \text { Value }=\{\text { error }, \text { null }\} \cup\{\text { true }, \text { false }\} \cup \mathbb{Z} \cup \text { ObjValue } \\
& \text { ObjValue }=\{f:(\{\text { myclass }\} \cup \text { LName }) \pitchfork(\text { CName } \cup \text { Value }) \\
& \text { myclass } \in \operatorname{dom} f \wedge f \text { myclass } \in \text { CName } \wedge \\
& (\{\text { myclass }\} \varangle f) \subseteq(\text { LName } \pitchfork \text { Value })\}
\end{aligned}
$$

Values for other primitive types should also be included. An object value is a mapping from field names to values, with the distinguished name myclass mapped to a class name.

The meanings of data types are parameterized by a typing environment. For primitives, we define $\llbracket$ bool $\rrbracket_{\Gamma}=\{$ error, true, false $\}$ and $\llbracket \mathbf{i n t} \rrbracket_{\Gamma}=\{$ error $\} \cup \mathbb{Z}$. For $N$ in $\Gamma$.cnames, we define $\llbracket N \rrbracket_{\Gamma}$ to be the correctly-typed object values.

$$
\begin{aligned}
\llbracket N \rrbracket_{\Gamma}= & \{\text { error, null }\} \cup \\
& \{f: \text { ObjValue } \mid \\
& \operatorname{dom} f=\operatorname{dom}(\Gamma \text {.attr }(f \text { myclass })) \cup\{\text { myclass }\} \wedge \\
& f \text { myclass } \leq_{\Gamma} N \wedge \\
& \left.\forall x: \operatorname{dom}(\Gamma \text {.attr }(f \text { myclass })) \bullet f x \in \llbracket \Gamma \text { attr }(f \text { myclass }) x \rrbracket_{\Gamma}\right\}
\end{aligned}
$$

It is straightforward to prove that $N \leq_{\Gamma} N^{\prime}$ implies $\llbracket N \rrbracket_{\Gamma} \subseteq \llbracket N^{\prime} \rrbracket_{\Gamma}$.

States are elements of ObjValue, although the "attributes" in a state include values of parameters and local variables. We write $\llbracket \Gamma, N \rrbracket$ for the set of states for class $N$ and typing environment $\Gamma$. An state $\sigma$ is in $\llbracket \Gamma, N \rrbracket$ just if it satisfies the following conditions. First, $\sigma$ gives values to the attributes of the actual class, if it is not main, and to the variables in $\Gamma$.locals.

$$
N \neq \operatorname{main} \Rightarrow \operatorname{dom} \sigma \backslash\{\text { myclass }\}=\operatorname{dom}(\Gamma \text {.attr }(\sigma \text { myclass })) \cup \operatorname{dom}(\Gamma \text {.locals })
$$

The union is not disjoint: $\Gamma$.locals declares the visible attributes and any local variables and method parameters; $\Gamma$. $\operatorname{attr}(\sigma$ myclass $))$ declares all attributes, 
including inherited private ones. If $N$ is main, $\sigma$ gives values just to the variables in $\Gamma$.locals. Also, if $N$ is not main, then myclass is a subclass of $N$; otherwise, myclass is main itself. The last condition is that $\sigma$ assigns values of the correct type. For $N \neq$ main and $x$ in $\operatorname{dom} \sigma \backslash\{$ myclass $\}$ we require $x \in \operatorname{dom}(\Gamma$.attr $N)$ to imply $\sigma x \in \llbracket \Gamma$.attr $N x \rrbracket_{\Gamma}$, and $x \in \operatorname{dom} \Gamma$.locals to imply $\sigma x \in \llbracket$. locals $x \rrbracket_{\Gamma}$. Just the latter implication applies if $N=$ main.

\subsection{Expressions and Predicates}

For $\eta \in \llbracket \Gamma \rrbracket, \sigma \in \llbracket \Gamma, N \rrbracket$, and derivable $\Gamma, N \triangleright e: T$, we define $\llbracket \Gamma, N \triangleright e: T \rrbracket \eta \sigma$, the value of $e$ in state $\sigma$. It is an element of $\llbracket T \rrbracket_{\Gamma}$ (Lemma 5).

We assume that for built-in function $f: T \rightarrow U$ a semantics is given, as a total function $\llbracket T \rrbracket_{\Gamma} \rightarrow \llbracket U \rrbracket_{\Gamma}$. The semantics of self is as follows.

$\llbracket \Gamma, N \triangleright$ self $: N \rrbracket \eta \sigma=(\{$ myclass $\} \cup \operatorname{dom}(\Gamma$.attr $(\sigma$ myclass $))) \triangleleft \sigma$

This uses domain restriction $(\triangleleft)$ of $\sigma$ : the attributes and myclass are retained; local variables and parameters are dropped. The similar definition for super and those for null and variables are omitted. We define $\llbracket \Gamma, N \triangleright$ new $N^{\prime}: N^{\prime} \rrbracket \eta \sigma$ as init $\Gamma N^{\prime}$ where init $\Gamma N^{\prime}$ is an object initialized with default values: false for boolean attributes, 0 for integers and null for objects. For other primitive types a default initial value should be given.

The value of the boolean expression $e$ is $N^{\prime \prime}$ is determined by whether the value of $e$ is an object of class $N^{\prime \prime}$. We omit the null and error cases.

$$
\frac{\llbracket \Gamma, N \triangleright e: N^{\prime} \rrbracket \eta=v \quad v \notin\{\text { null, error }\}}{\llbracket \Gamma, N \triangleright e \text { is } N^{\prime \prime}: \text { bool } \rrbracket \eta \sigma=\left(v \text { myclass } \leq_{\Gamma} N^{\prime \prime}\right)}
$$

Semantics of attribute selection, update, and cast are straightforward; they yield error for null.

The semantics $\llbracket \Gamma, N \triangleright \psi:$ pred $\rrbracket \eta$ of a predicate $\psi$ is a subset of $\llbracket \Gamma, N \rrbracket$ (Lemma 6). The semantics of expressions as formulas, and of the logical operations, is standard and omitted. The semantics of isExactly is similar to that of is.

$$
\llbracket \Gamma, N \triangleright e: N^{\prime} \rrbracket \eta=f
$$

$\llbracket \Gamma, N \triangleright e$ isExactly $N^{\prime \prime}: \operatorname{pred} \rrbracket \eta=$

$\left\{\sigma: \llbracket \Gamma, N \rrbracket \mid f \sigma \notin\{\right.$ null, error $\} \wedge(f \sigma)$ myclass $\left.=N^{\prime \prime}\right\}$

The coercion rules have similar semantics; we consider that involving is and $\wedge$.

$$
\llbracket\left(\Gamma ; x: N^{\prime \prime}\right), N \triangleright \psi: \operatorname{pred} \rrbracket \eta=\Sigma \quad N^{\prime \prime} \leq_{\Gamma} N^{\prime}
$$

$\llbracket\left(\Gamma ; x: N^{\prime}\right), N \triangleright x$ is $N^{\prime \prime} \wedge \psi:$ pred $\rrbracket \eta=$
$\left\{\sigma: \llbracket\left(\Gamma ; x: N^{\prime}\right), N \rrbracket \mid(\sigma x) \notin\{\right.$ null, error $\} \wedge(\sigma x)$ myclass $\left.\leq_{\Gamma} N^{\prime \prime} \wedge \sigma \in \Sigma\right\}$

This combines the interpretations of the combined operators. 


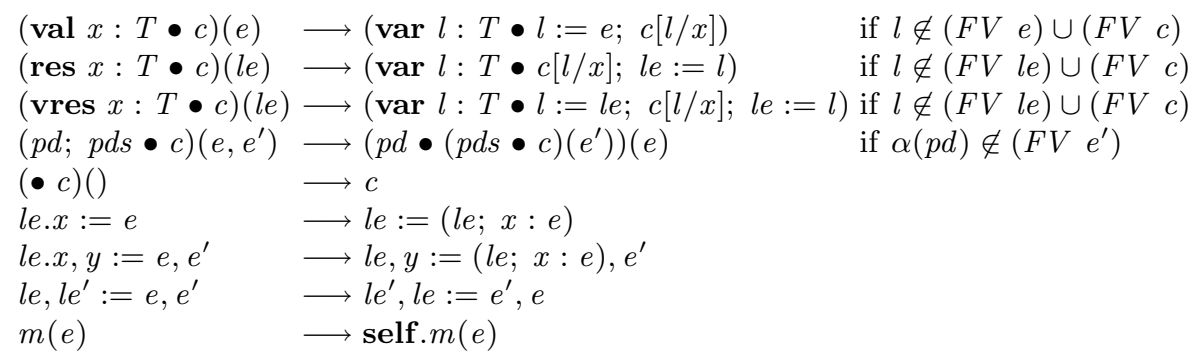

Table 4. Syntactic transformations

\subsection{Commands and Parameterized Commands}

For command typing $\Gamma, N \triangleright c$ : com and environment $\eta \in \llbracket \Gamma \rrbracket$, the semantics $\llbracket \Gamma, N \triangleright c: \mathbf{c o m} \rrbracket \eta$ is a total function on formulas (Theorem [1) which, when applied to a formula typable in $\Gamma, N$ yields a result typable in $\Gamma, N$ (Theorem 2).

Assignments to general left-expressions are dealt with using syntactic transformations that yield assignments of update expressions to simple variables and to self. Assignment to simple variables is interpreted using substitution.

$$
\frac{\Gamma \triangleright x: T \quad \Gamma \triangleright e: T^{\prime} \quad T^{\prime} \leq_{\Gamma} T}{\llbracket \Gamma \triangleright x:=e: \operatorname{com} \rrbracket \eta \psi=(e \neq \text { error } \wedge \psi[e / x])}
$$

We use an expression "error". In this paper we omit error from the grammar because it has no other use; its typing rule and semantics are straightforward.

User programs should not include assignments to self and method calls where self is used as a result or value-result argument. Assignments to self are introduced only in the syntactic transformations for parameter passing, when the argument corresponding to the me parameter of a method is self. This guarantees that self is always assigned an object of the current class, but the semantics cannot depend on this assumption.

$$
\Gamma, N \triangleright e: N^{\prime} \quad N^{\prime} \leq_{\Gamma} N
$$

$$
\begin{aligned}
& \llbracket \Gamma, N \triangleright \text { self }:=e: \operatorname{com} \rrbracket \eta \psi= \\
& \left(\vee_{N^{\prime} \leq_{\Gamma} N}^{\bullet} e \text { isExactly } N^{\prime} \wedge \psi[e, e . x / \text { self }, x]\right) \text { where } x=\operatorname{dom}\left(\Gamma \text {.attr } N^{\prime}\right)
\end{aligned}
$$

This uses a disjunction over the subclasses $N^{\prime}$ of $N$; each disjunct involves a substitution for appropriate attributes. There is no need to check that $e$ is not error because error isExactly $N^{\prime}$ is false, for all $N^{\prime}$. If the only assignments to self are those introduced in the semantics, self is always assigned an object of the current class, in which case the semantics simplifies to $\psi[e, e . x /$ self,$x]$. We need not give an operational justification for the general case.

We define $\llbracket \Gamma ; x: T \triangleright x:\left[\psi_{1}, \psi_{2}\right]: \mathbf{c o m} \rrbracket \eta \psi$ to be $\psi_{1} \wedge\left(\forall x: T \bullet \psi_{2} \Rightarrow \psi\right)$ as in Morgan's work. We also use the standard semantics for control constructs and blocks. 
Parameter passing and various forms of assignment are reduced by the rule below to more basic constructs using the relation $\longrightarrow$ defined in Table 4 .

$$
\frac{c \longrightarrow{ }^{*} c^{\prime} \quad \llbracket \Gamma, N \triangleright c^{\prime}: \mathbf{c o m} \rrbracket \eta=g}{\llbracket \Gamma, N \triangleright c: \mathbf{c o m} \rrbracket \eta=g}
$$

If $\Gamma, N \triangleright c: \mathbf{c o m}$ and $c \longrightarrow c^{\prime}$ then $\Gamma, N \triangleright c^{\prime}: \mathbf{c o m}$ (Lemma 3). The reflexivetransitive closure $\longrightarrow$ * $\longrightarrow$ reduces every derivable command typing to one for which there is a direct semantic definition (Theorem 11). The first five transformations rewrite parameter passing in the usual way; $\alpha(p d)$ denotes the set of variables declared in $p d$, and $F V$ gives free variables. The next three transformations rewrite assignments to left-expressions into assignments to simple variables or self. The last transformation inserts the missing object (self) in a method call $m(e)$.

\subsection{Programs and Method Calls}

The meaning of a complete program is the meaning of its main command, in an appropriate environment. The typing includes global variables $x$ of $c$.

$$
\begin{gathered}
\llbracket \Gamma, \text { main } \triangleright c: \mathbf{c o m} \rrbracket \eta=f \quad \Gamma=((\text { VDecs cds main }) ; x: T) \\
\text { Vmeth } \Gamma c d s \quad \eta=\text { Meths } \Gamma c d s \\
\llbracket \varnothing ; x: T \triangleright c d s \bullet c: \text { program } \rrbracket=f
\end{gathered}
$$

The environment $\eta$ records the methods available for objects of each of the classes declared in $c d s$; these methods are extracted from $c d s$ by the function Meths which builds $\eta$ as follows.

For each class $N$ and method $m$, the parameterized command $\eta N m$ has an extra value-result parameter $m e$, and in its body each occurrence of an attribute $x$ of $N$ or of a call to a method $m$ of $N$ is replaced by me. $x$ and me.m. Only "top level" occurrences of attributes are changed: if $x$ is an attribute, then $x . x$ becomes me.x.x. For a class that inherits $m$, me must be given the more specific type; it always has exactly the type of the object, compatible with the typing rule for value-result parameters.

If the declared body of a method $m$ contains recursive invocations, then $\eta N m$ is the least fixed point of the context determined by the body. This approach is also used in Back's work and CSW98 to deal with recursive parameterized procedures. We forbid mutual recursion so that fixpoints can be taken separately for each method. We justify existence of the least fixed point by techniques used in the cited works; it depends on monotonicity (Theorem 3 ).

Finally we consider method calls le.m(e). Even though le.m is a parameterized command, typed for example as $\Gamma, N \triangleright l e . m: \operatorname{pcom}(\operatorname{val} x: T)$, no transformation rule is applicable. In a state where the dynamic type of $l e$ is $N^{\prime}, \eta N^{\prime} m$ takes the form (vres $m e: N^{\prime} ;$ val $x: T \bullet c$ ), and if we define $f_{N^{\prime}}$ as $\llbracket \Gamma, N \triangleright\left(\right.$ vres $m e: N^{\prime} ;$ val $\left.x: T \bullet c\right)(l e, e): \mathbf{c o m} \rrbracket \eta$, then we should define $\llbracket \Gamma, N \triangleright \operatorname{le} . m(e): \mathbf{c o m} \rrbracket \eta \psi$ to be $f_{N^{\prime}} \psi$. The semantics of method call is the 
disjunction, over the possible classes $N^{\prime}$, of le isExactly $N^{\prime} \wedge f_{N^{\prime}} \psi$. Thus the semantics $f_{N^{\prime}}$ is used just when it should be. The possible classes $N^{\prime}$ are the subclasses of the static type $N^{\prime \prime}$ of le, determined by the derivation of le.

$$
\frac{\llbracket \Gamma, N \triangleright\left(\eta N^{\prime} m\right)(l e, e): \mathbf{c o m} \rrbracket \eta=f_{N^{\prime}} \quad \text { all } N^{\prime} \leq_{\Gamma} N^{\prime \prime}, \text { for } N^{\prime \prime} \text { the type of } l e}{\llbracket \Gamma, N \triangleright l e . m(e): \mathbf{c o m} \rrbracket \eta \psi=\left(\vee_{N^{\prime} \leq_{\Gamma} N^{\prime \prime}} \bullet l e \text { isExactly } N^{\prime} \wedge f_{N^{\prime}} \psi\right)}
$$

The hypothesis depends on $\eta N^{\prime} m$ being typable in $\Gamma, N$. The free variables in the original declaration of $m$ are attributes visible in the class, now accessed through the me parameter. Those attributes are not necessarily visible in the context of the call, so references me.x are only typable in the extended system.

\subsection{Example}

The program below acts on a global variable $c$ of type $C$. For clarity, we write the body of a method with no parameters as a command, instead of as a parameterized command with an empty declaration.

class $C$ pri $x:$ int; meth $\operatorname{Inc} \widehat{=} x:=x+1 ;$ meth $D e c \widehat{=} x:=x-1$ end - $\operatorname{c.} \operatorname{Inc}()$

We calculate the weakest precondition for this program to establish c. $x>0$. Writing $C D$ to stand for the declaration of $C$ above, we begin.

$$
\begin{aligned}
& \llbracket \varnothing ; c: C ; \triangleright C D \bullet c \cdot \operatorname{In} c(): \text { program } \rrbracket(c . x>0) \\
& =\llbracket \Gamma \text {, main } \triangleright c . \operatorname{In} c(): \mathbf{c o m} \rrbracket \eta(c . x>0)
\end{aligned}
$$

Here the typing environment $\Gamma=($ VDecs $C D$ main); $c: C$ is as follows.

$$
\begin{aligned}
& (\text { attr }=\{\text { object } \mapsto \varnothing, C \mapsto\{x \mapsto \text { int }\}\}, \\
& \text { meth }=\{\text { object } \mapsto \varnothing, C \mapsto\{\text { Inc } \mapsto \varnothing, D e c \mapsto \varnothing\}\}, \\
& \text { vis }=\{\text { object } \mapsto \varnothing, C \mapsto\{x \mapsto \text { pri }\}\}, \\
& \text { cnames }=\{\text { object }, C\}, \text { supcls }=\{C \mapsto \text { object }\}, \text { locals }=\{c \mapsto C\})
\end{aligned}
$$

The environment $\eta=$ Meth $\Gamma C D$ is shown below.

$\{$ object $\mapsto \varnothing, C \mapsto\{$ Inc $\mapsto($ vres me $: C \bullet$ me.x $:=m e . x+1)$, Dec $\mapsto \ldots\}\}$

We proceed as follows.

$$
\begin{aligned}
& \llbracket \Gamma, \text { main } \triangleright c . \operatorname{Inc}(): \mathbf{c o m} \rrbracket \eta(c . x>0) \\
& =\left(\vee_{N^{\prime} \leq_{\Gamma} C} \bullet c \text { isExactly } N^{\prime} \wedge \llbracket \Gamma, \text { main } \triangleright\left(\eta N^{\prime} \text { Inc }\right)(c): \mathbf{c o m} \rrbracket(c . x>0)\right) \\
& \quad[\text { by the semantics of method call] } \\
& =c \notin\{\text { null, error }\} \wedge \llbracket \Gamma, \text { main } \triangleright(\eta C \text { Inc })(c): \mathbf{c o m} \rrbracket(c . x>0) \\
& \quad[\text { by } C \text { has no proper subclasses and the semantics of isExactly] } \\
& =c \notin\{\text { null, error }\} \wedge \\
& \quad \llbracket \Gamma, \text { main } \triangleright(\text { vres the definition of } \eta]
\end{aligned}
$$




$$
\begin{array}{rlr}
= & c \notin\{\text { null, error }\} \wedge & \text { [by a syntactic transformation] } \\
& \llbracket \Gamma, \text { main } \triangleright(\operatorname{var} l: C \bullet l:=c ; l . x:=l . x+1 ; c:=l): \mathbf{c o m} \rrbracket \eta(c . x>0) \\
= & c \notin\{\text { null, error }\} \wedge \quad \text { [by the semantics of variable blocks] } \\
& \forall l \bullet \llbracket \Gamma ; l: C, \text { main } \triangleright(l:=c ; l . x:=l . x+1 ; c:=l): \text { com } \rrbracket \eta(c . x>0) \\
= & c \notin\{\text { null, error }\} \wedge c . x \neq \text { error } \wedge(c . x>0)[l / c][(l ; x: l . x+1) / l][c / l]
\end{array}
$$

[by the semantics of sequence and assignment]

$=c \notin\{$ null, error $\} \wedge c . x \neq$ error $\wedge c . x+1>0$

[by a properties of substitution and update expressions]

The result obtained is exactly what should be expected.

\section{Properties of the Semantics}

This section shows that the semantics is a well-defined function of typings, and that it is type-correct. Before presenting these theorems, however, we present auxiliary results.

Lemma 3. The syntactic transformations preserve typing, in the sense that $\Gamma, N \triangleright c: \mathbf{c o m}$ and $c \longrightarrow c^{\prime}$ imply $\Gamma, N \triangleright c^{\prime}: \mathbf{c o m}$, for all $c, c^{\prime}$.

To prove the type-correctness theorem, we need typability to be preserved by substitution on formulas. This result holds only in the extended type system, where subtyping constraints are dropped from the rules for type tests and casts.

Lemma 4. (a) Suppose $\Gamma, N \triangleright \psi$ : pred is derivable and $x$ is free in $\psi$; let $T$ be the type of $x$ (which is uniquely determined by $\Gamma, N)$. If $T^{\prime} \leq_{\Gamma} T$ and $\Gamma, N \triangleright e: T^{\prime}$ is derivable then $\Gamma, N \triangleright \psi[e / x]$ : pred is derivable. (b) Same as part (a) but with self in place of $x$.

The rules for assignment and result-parameter passing also involve subtyping constraints, but that does not invalidate Lemma 4 because predicate typings do not depend on command typings.

Because the semantics of ROOL is not defined by structural recursion on program texts, we need to show that the notation is coherent, in the sense that $\llbracket \Gamma, N \triangleright$ phrase $: \theta \rrbracket$ is a function of the typing $\Gamma, N \triangleright$ phrase $: \theta$. Expression typings have unique derivations (Lemma 1), and the semantics is defined directly in terms of the typing rules, so coherence for expressions is immediate. As a result, type-correctness for expressions is straightforward.

Lemma 5. If $\Gamma, N \triangleright e: T$ then $\llbracket \Gamma, N \triangleright e: T \rrbracket \eta \sigma \in \llbracket T \rrbracket_{\Gamma}$ for all $\eta \in \llbracket \Gamma \rrbracket$ and $\sigma \in \llbracket \Gamma, N \rrbracket$.

Due to the coercion rules, predicate typings are not unique. We need a coherence lemma. 
Lemma 6. The semantics $\llbracket \Gamma, N \triangleright \psi$ : pred $\rrbracket$ of a predicate typing is a function of the typing $\Gamma, N \triangleright \psi$ : pred, and $\llbracket \Gamma, N \triangleright \psi:$ pred $\rrbracket \subseteq \llbracket \Gamma, N \rrbracket$.

For command typings, derivations are unique except for derivations of predicates that occur within commands. Nevertheless, the semantics of commands does not depend on semantics of predicates, so there is no issue of coherence.

There are two parts of the semantics of commands, however, that are not simply defined by structural recursion on derivations. The first is that for some commands the semantics is given indirectly by syntactic transformation. Nonetheless, these transformations preserve typing (Lemma 3), and the derivations of the transformed phrases are built from the derivations of the original phrases in such a way that the semantics depends only on the semantics of subderivations.

Method calls are the second difficult part: $\llbracket \Gamma, N \triangleright l e . m(e): \mathbf{c o m} \rrbracket \eta$ depends on the semantics of method calls $\llbracket \Gamma, N \triangleright \eta N^{\prime} m(e)$ : com $\rrbracket \eta$ where $N^{\prime}$ ranges over subtypes of the type $N^{\prime \prime}$ of le. The parameterized command $\eta N^{\prime} m$ can contain method calls, so the semantics of a method call depends on the semantics of method calls, which are certainly not part of the derivation of le.m(e).

However, we are only concerned recursion-free environments: those obtained from Meth $\Gamma$ cds, in which recursion has been resolved already. The semantics of a method $m$ of a class $N$ depends only on methods $N^{\prime}, m^{\prime}$ that do not depend on $N, m$, and the relation "can call" on pairs $N^{\prime}, m^{\prime}$ is well founded. We combine this lexicographically with the order "is a subderivation" to obtain a well founded order. We define the notion of the semantics $\llbracket \Gamma, N \triangleright$ phrase $: \theta \rrbracket$ in the context of some method $N^{\prime}, m^{\prime}$; this depends on subderivations of phrase : $\theta$ and also on semantics for phrases in context of methods $N^{\prime \prime}, m^{\prime \prime}$ smaller than $N^{\prime}, m^{\prime}$.

Theorem 1. For all derivable $\Gamma, N \triangleright c$ : com and all $\eta \in \llbracket \Gamma \rrbracket$, the semantics $\llbracket \Gamma, N \triangleright c: \mathbf{c o m} \rrbracket \eta$ is a total function on all formulas, regardless of type, provided that $\eta$ is recursion-free.

Proof By induction with respect to the order discussed above.

Case assignment: for assignments to simple identifiers, and for assignments to self, the semantics is given directly. Others are reduced by syntactic transformations to the simple case. By Lemma 3 the transformed assignments are typable in $\Gamma, N$. Any assignment can be rewritten to a simple one which is unique up to the order in which variables are listed; and order does not affect the semantics. Case specification statement: this has a single typing rule and the semantics is given directly.

Case application $p c(e)$ of an explicit parameterized command (not a method call): the transformation rules eliminate $\operatorname{argument}(\mathrm{s}) e$ in favor of local variables and assignments. The result is typable (Lemma 3). Moreover, the derivation of the transformed command is composed of subderivations of the original command. Introducing local variables involves the choice of identifier $l$, but the semantics is independent of the choice because $l$ is bound by $\forall$.

Case method call applied to parameters: a method call $m(e)$ is reduced to self. $m(e)$, which has the general form $l e . m(e)$. Let $\psi$ be any formula. The semantics for le.m(e) is defined provided each $f_{N^{\prime}}$, i.e. $\llbracket \Gamma, N \triangleright \eta N^{\prime} m(l e, e): \mathbf{c o m} \rrbracket \eta$, 
is defined. By the conditions on environments, $\eta N^{\prime} m(l e, e)$ is typable. The methods on which $\eta N^{\prime} m$ depends are smaller in our ordering, by the proviso that $\eta$ is recursion-free. By induction, $\llbracket \Gamma, N \triangleright \eta N^{\prime} m(l e, e): \mathbf{c o m} \rrbracket \eta$ denotes a total function on formulas, and hence so does the semantics of the call.

Cases explicit recursion: this is defined using least fixpoints of program contexts. Because these are monotonic (Theorem 3), the least fixpoints are well defined.

Cases sequence, alternation and variable blocks: in each case there is a direct semantic definition and the result holds by induction.

Theorem 2. If $\Gamma, N \triangleright \psi$ : pred and $\Gamma, N \triangleright c$ : com are derivable then so is $\Gamma, N \triangleright(\llbracket \Gamma, N \triangleright c: \mathbf{c o m} \rrbracket \eta \psi)$ : pred, provided $\eta$ is recursion-free.

Proof By induction, using the order defined above.

Case assignment: for simple variables, the semantics requires that the predicate $e \neq$ error $\wedge \psi[e / x]$ be typable in $\Gamma$ provided that $\psi$ is. Thus we need that $\Gamma \triangleright x: T$ and $\Gamma \triangleright e: T^{\prime}$ and $T^{\prime} \leq_{\Gamma} T$ imply $\Gamma \triangleright \psi[e / x]:$ pred. That is by Lemma 4(a). To type $e \neq$ error, we use the typing rule for error (which gives it any type), and then the rule for built-in functions to type the equality. For assignments to self, suppose $\psi$ is typable in $\Gamma, N$. For each $N^{\prime}$, we have, by Lemma 4(b), $\psi[e, e . x /$ self, $x]$ typable in $\Gamma, N^{\prime}$. Moreover, if an assignment to self is typable in $\Gamma, N$, then self is typable in $\Gamma, N$ and so $N \neq$ main. Thus, by Lemma 2, $\left(\vee_{N^{\prime} \leq{ }_{\Gamma} N} \bullet\right.$ self isExactly $N^{\prime} \wedge \psi[e, e . x /$ self, $\left.x]\right)$ is typable in $\Gamma, N$. Case specification statement: for $\Gamma ; x: T \triangleright x:\left[\psi_{1}, \psi_{2}\right]$ : com to be derivable, $\psi_{1}$ and $\psi_{2}$ are typable in $\Gamma ; x: T$. For $\psi$ with $\Gamma ; x: T \triangleright \psi:$ pred the semantics yields $\psi_{1} \wedge\left(\forall x: T \bullet \psi_{2} \Rightarrow \psi\right)$, which can be typed for $\Gamma ; x: T$ using the rules for $\wedge, \forall$, and $\Rightarrow$.

Cases sequence: straightforward use of induction.

Case alternation: by induction, each $f_{i}$ in the semantics yields well-typed formulas, and the guards have to be typable predicates in $\Gamma, N$, so the formula $\left(\vee i \bullet \psi_{i}\right) \wedge\left(\wedge i \bullet \psi_{i} \Rightarrow f_{i} \psi\right)$ is also typable using the rules for $\wedge, \vee$, and $\Rightarrow$.

Case method call: for method calls le.m(e), we have to show that the predicate $\left(\vee_{N^{\prime}} \bullet\right.$ le isExactly $\left.N^{\prime} \wedge f_{N^{\prime}} \psi\right)$ is typable in $\Gamma, N$. By induction, each $f_{N^{\prime}}$ applies to formulas typable in $\Gamma, N$, and each returns the same. Now le is typable in $\Gamma, N$, so by using the rules $\vee, \wedge$, and isExactly we obtain the desired result. Case blocks: the weakest precondition $\llbracket \Gamma \triangleright(\operatorname{var} x: T \bullet c$ end $): \operatorname{com} \rrbracket \eta \psi$ is defined as $(\forall x: T \bullet f \psi)$, where $f=\llbracket \Gamma ; x: T \triangleright c: \mathbf{c o m} \rrbracket \eta$. If $\psi$ is typable in $\Gamma$ then it is also typable in $\Gamma ; x: T$. Therefore $f$ can be applied to $\psi$ and by induction $f \psi$ is typable in $\Gamma ; x: T$, and hence by the typing rule for $\forall$ we get $(\forall x: T \bullet f \psi)$ typable in $\Gamma$. Similar considerations apply to avar blocks.

It is straightforward to formulate and prove definedness and type-preservation for complete programs, using Theorems 1 and 2 .

\section{Refinement}

In this section we define notions of refinement and give the basic result on monotonicity. To simplify definitions, we assume that all phrases are well-typed. 
The fundamental refinement relationship $\sqsubseteq$ is between programs. This is based on pointwise order on predicate transformers, as usual, but restricted to healthy predicates just as in languages where procedures can be assigned to variables [Nau98b, HH98]. As an example, if class CPt suitably refines Pt we expect the refinement $x:=$ new $P t \sqsubseteq x:=$ new $C P t$. But the postcondition $x$ isExactly $P t$ is established only by the first assignment. The solution is to restrict attention to monotonic predicates. For our purposes, a predicate $\psi$ is monotonic provided that for any object values ov 1 , ov 2 , if ov 1 satisfies $\psi$ and ov 2 myclass $\leq_{\Gamma}$ ov 1 myclass, and ov 2 agrees with ov 1 on all the attributes of ov 1 myclass, then ov 2 satisfies $\psi$.

Definition 1. For sequences of class declarations $c d s$ and $c d s^{\prime}$, commands $c$ and $c^{\prime}$ with the same free variables $x: T$, define $(c d s \bullet c) \sqsubseteq\left(c d s^{\prime} \bullet c^{\prime}\right)$ if and only if, for all monotonic $\psi$,

$$
\llbracket \varnothing ; x: T \triangleright(c d s \bullet c): \operatorname{program} \rrbracket \psi \Rightarrow \llbracket \varnothing ; x: T \triangleright\left(c d s^{\prime} \bullet c^{\prime}\right): \operatorname{program} \rrbracket \psi
$$

The free variables of a program represent its input and output; therefore, it makes sense to compare only programs with the same free variables.

A program can be refined by refining its command part and its class declarations. Commands in ROOL apear in the context of a sequence of class declarations, so we first define relation $c d s, N \triangleright c \sqsubseteq c^{\prime}$, which establishes that in the context of $c d s$ the command $c$ occurring in the class $N$ is refined by $c^{\prime}$.

Definition 2. For a sequence of class declarations $c d s$, commands $c$ and $c^{\prime}$, and a class $N$, define cds, $N \triangleright c \sqsubseteq c^{\prime}$ if and only if, for all monotonic predicates $\psi$,

$$
\llbracket \Gamma, N \triangleright c: \mathbf{c o m} \rrbracket \eta \psi \Rightarrow \llbracket \Gamma, N \triangleright c^{\prime}: \mathbf{c o m} \rrbracket \eta \psi
$$

where $\Gamma=($ VDecs cds $N) ; x: T, x$ are the method parameters and local variables in scope, and $\eta=$ Meths $\Gamma$ cds.

Because methods are parameterized commands, we need the analog of Definition 1 for them.

Definition 3. For sequence of class declarations cds, parameterized commands $p c$ and $p c^{\prime}$, which have the same parameters, and a class $N, c d s, N \triangleright p c \sqsubseteq p c^{\prime}$ if and only if, for all (lists of) expressions $e, c d s, N \triangleright p c(e) \sqsubseteq p c^{\prime}(e)$

This is a straightforward extension Back's definition (see [CSW98]).

Using induction as in Theorems 1 and 2, the following can be proved.

Theorem 3. Suppose we have a sequence of class declarations cds, a class $N$, a parameterized command $p c$, and a context $\mathcal{C}[\cdot]$ which is a parameterized command, and so, a function from parameterized commands to parameterized commands. If we have that $c d s, N \triangleright p c \sqsubseteq p c^{\prime}$, then $c d s, N \triangleright \mathcal{C}[p c] \sqsubseteq \mathcal{C}\left[p c^{\prime}\right]$. Similarly, the command constructors are monotonic.

This theorem justifies our treatment of recursion and recursive methods. 
As a class is a data type, refinement of classes is related to data refinement HHS87. We define the relation view, $c d s \triangleright c d s^{\prime} \preccurlyeq c d s^{\prime \prime}$, for a list of methods view and sequences of class declarations $c d s, c d s^{\prime}$, and $c d s^{\prime \prime}$. The meaning is that in the context of $c d s$, if only methods listed in view are used, then the class declaration $c d s^{\prime}$ can be replaced by $c d s^{\prime \prime}$.

Definition 4. For a list of methods view, sequences of class declarations cds, $c d s^{\prime}$, and $c d s^{\prime \prime}$, view, $c d s \triangleright c d s^{\prime} \preccurlyeq c d s^{\prime \prime}$ if and only if, for all commands $c$ that uses only methods in view, $\left(c d s ; c d s^{\prime} \bullet c\right) \sqsubseteq\left(c d s ; c d s^{\prime \prime} \bullet c\right)$.

Refinement between single classes $c d^{\prime}$ and $c d^{\prime \prime}$ is a special case. By considering a more general relation, we allow for restructuring a collection of class declarations. In practice, Definition 4 would not be used directly, but it is the fundamental notion with respect to which techniques such as downward and upward simulation must be proved sound HHS87, Nau98a].

\section{Discussion}

We have shown how the standard predicate-transformer model can be extended to an object-oriented language. The semantics can be modified to allow arbitrary mutual recursion among methods, at the cost of taking a single fixpoint for the entire environment of methods. This makes it more complicated to prove refinement laws, so we have chosen the simpler approach at this stage.

Others [Lei98, MS97 BKS98] have extended existing refinement calculi with object-oriented features, but restricting inheritance or not dealing with classes and visibility. Those works, however, deal with sharing and concurrency. Another approach to objects is implicit in the parametricity semantics of Algol-like languages. It has been adapted to object-oriented programs by Reddy Red98, with whom we are collaborating to give a semantics for ROOL.

The main shortcoming of our semantics is that it is not entirely compositional. Since our aim is to validate laws like those in [Bor98], for when one class is a behavioural subclass of another, within the context of some other classes, this is a potential problem. However, the touchstone criteria for behavioural refinement is that $c d s_{1} \bullet c \sqsubseteq c d s_{2} \bullet c$ should hold whenever $c d s_{2}$ is obtained from $c d s_{1}$ by behavioural refinement of some classes. Fortunately, this has a natural formulation with a single context that includes all relevant classes.

Our notion of class refinement corresponds to the notion of behavioural subtyping introduced by Liskov and Wing [LW94. Definition 4 captures the essence of their subtype requirement. In our framework the property of interest is refinement of programs, which captures the notion of total correctness. The two ways of defining the subtype relation presented in [LW94] are based on the downward simulation technique HHS87], specialized to the particular case of functional data refinement. We expect that particular techniques like these can be proved sound with respect to Definition 4. Similarly, Liskov and Wing claim, but do not formalize, that their definitions satisfy the subtype requirement. 
By using a language of specification and programming, we do not need a distinction between specifications and implementations of classes. As already seen in traditional refinement calculi, this simplifies both the theory of refinement and the presentation and application of refinement laws.

Acknowledgement This work benefitted from discussions with our collaborators Augusto Sampaio, Uday Reddy, Paulo Borba, and Hongseok Yang. UFPE and Stevens provided generous support for travel.

\section{References}

[AdB94] Pierre America and Frank de Boer. Reasoning about dynamically evolving process structures. Formal Aspects of Computing, 6:269-316, 1994.

[AL97] Martín Abadi and K. Rustan Leino. A logic of object-oriented programs. In Proceedings, TAPSOFT 1997. Springer-Verlag, 1997. Expanded in DEC SRC report 161.

[BvW98] R. J. R. Back and J. von Wright. Refinement Calculus: A Systematic Introduction. Graduate Texts in Computer Science. Springer-Verlag, 1998.

[BKS98] Marcello M. Bonsangue, Joost N. Kok, and Kaisa Sere. An approach to object-orientation in action systems. In Johan Jeuring, ed., Mathematics of Program Construction, LNCS 1422, pages 68-95. Springer, 1998.

[Bor98] Paulo Borba. Where are the laws of object-oriented programming? In $I$ Brazilian Workshop on Formal Methods, pages 59-70, Porto Alegre, Brazil, 19th-21st October 1998.

[CN99] A. L. C. Cavalcanti and D. A. Naumann. A Weakest Precondition Semantics for an Object-oriented Language of Refinement - Extended Version. Available at http://www.di.ufpe.br/ ${ }^{\sim}$ alcc

[CSW98] A. L. C. Cavalcanti, A. C. A. Sampaio, and J. C. P. Woodcock. Procedures and Recursion in the Refinement Calculus. Journal of the Brazilian Computer Society, 5(1):1-15, 1998.

[CSW99] A. L. C. Cavalcanti, A. Sampaio, and J. C. P. Woodcock. An inconsistency in procedures, parameters, and substitution in the refinement calculus. Science of Computer Programming, 33(1):87-96, 1999.

[HH98] C. A. R. Hoare and J. He. Unifying Theories of Programming. Prentice Hall, 1998.

[HHS87] C. A. R. Hoare and J. He and J. W. Sanders. Prespecification in data refinement. Information Processing Letters, 25(2), 1987.

[Lan95] Kevin Lano. Formal Object-Oriented Development. Springer, 1995.

[Lei98] K. Rustan M. Leino. Recursive object types in a logic of object-oriented programming. In Chris Hankin, ed., 7th European Symposium on Programming, LNCS 1381. Springer, 1998.

[LW94] Barbara H. Liskov and Jeannette M. Wing. A behavioral notion of subtyping. ACM Transactions on Programming Languages and Systems, 16(6), 1994.

[MS97] A. Mikhajlova and E. Sekerinski, Class refinement and interface refinement in object-oriented programs. In Proceedings of FME'97: Industrial Benefit of Formal Methods. Springer, 1997.

[Mor94] Carroll Morgan. Programming from Specifications, 2ed. Prentice Hall, 1994. 
[Nau98a] David A. Naumann. Validity of data refinement for a higher order imperative language. Submitted.

[Nau98b] David A. Naumann. Predicate transformer semantics of a higher order imperative language with record subtypes. Science of Computer Programming, 1998. To appear.

[Red98] U. S. Reddy. Objects and classes in Algol-like languages. In Fifth Intern. Workshop on Foundations of Object-oriented Languages. URL: http://pauillac.inria.fr/ remy/fool/proceedings.html, Jan 1998. 